\title{
Some axially symmetric contact problems
}

\author{
Colin Atkinson
}

Received: 14 November 2018 / Accepted: 15 April 2019 / Published online: 21 May 2019

(C) The Author(s) 2019

\begin{abstract}
Axisymmetric stress analysis problems are considered where mixed boundary conditions apply over the interior of a cylinder. A brief indication of some generalisations is also given.
\end{abstract}

Keywords Axisymmetric contact problems · Mixed boundary conditions · Wellbore stress analysis

\section{Introduction}

In this paper we will discuss some axisymmetric stress analysis problems where there is contact along an inner region of a cylindrical boundary and hence result in mixed boundary conditions (which might be non-linear) on the inner boundary wall. The unmixed problem of a stress condition applied to the exterior wall of a finite cylinder was considered long ago by Tranter and Craggs [1] . For the current analysis the boundary might be the interior of a wellbore where for example packers (inflatable balloons made from elastomers reinforced by cords) are used by the oil industry for sealing an interval along the wellbore. This might be used for open hole stress tests to be performed. Such stress tests require that the pressure exerted by the packer on the formation needs to be as close as possible to the pressure in the interval to avoid fracture initiation or fracture growth along the packers. In addition axial tension in the rock needs to be kept to a minimum to avoid initiation of transverse fractures at the packer level. This is discussed in Atkinson et al. [2] where the borehole is considered to be rigid as far as the packer shape is concerned. As the pressure inside the packer increases, the balloon expands to flatten against the wall and the extra pressure beyond the touch pressure will cause stresses in the formation. The packer deformation is quite complicated as it flattens against the wall and is based on a theory due to Kydoniefs [3] and implemented by Atkinson and Peltier [4] who were also interested in the design of a reinforced packer. In this rock mechanics application the stress analysis reduces to a stress boundary condition on the wellbore which can be treated by Fourier transforming the boundary even if the rock were considered to be poroelastic. This stress analysis was also compared to finite element and various cases considered, including dual packer arrangements and different slip conditions at the wall. The lack of a displacement condition at the borehole wall and the approximation of a rigid boundary as a boundary condition for the expanding packer is accurate as far as this application is concerned. There are, of course, many other applications of the mechanics of poroelastic media, a recent example is Selvadurai and Suvorov [5]. However there are also many

C. Atkinson $(\varangle)$

Department of Mathematics, Imperial College, South Kensington, London SW7 2BZ, UK

e-mail: c.atkinson@ic.ac.uk 
Fig. 1 Model reduction framework

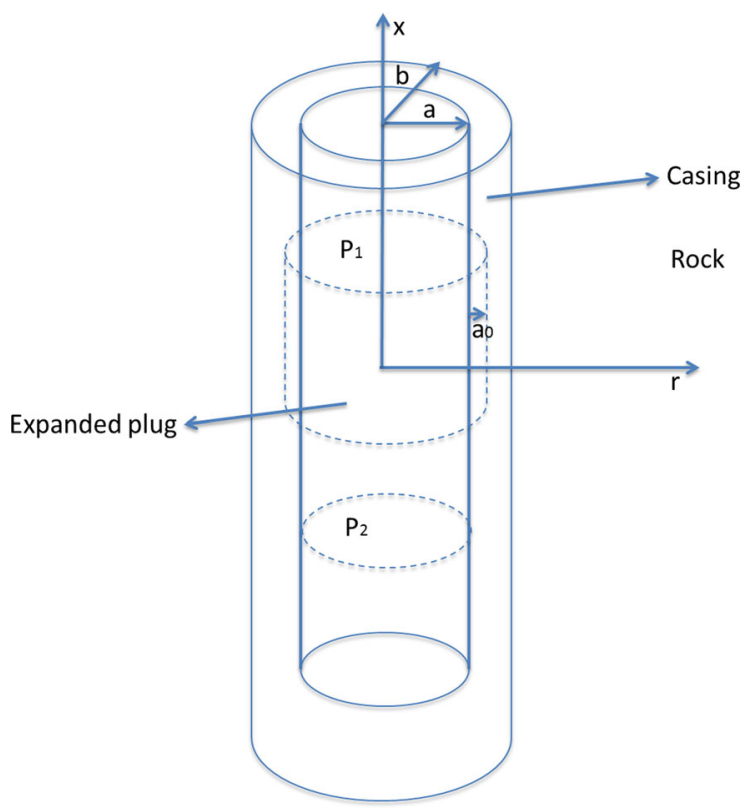

other situations where the expanding object has a more complicated interaction with a boundary region for example balloon angioplasty such as modelled by Eftaxiopoulos and Atkinson [6]. We use this as motivation to consider axisymmetric boundaries where the boundary conditions are mixed, i.e. stress boundary conditions in one region and displacement conditions in another. We also consider briefly a generalisation to more complicated conditions. We return to these problems later.

\section{Specified displacement over a region}

We begin by discussing the situation where a plug is effected in a wellbore by first placing it at a given location and then thermally expanding it into the casing by first melting the plug and subsequently merging into the metal casing or in principle into the rock if the wellbore were uncased (see e.g. Fig. 1).

We will assume here that the effect of this expansion is to cause a fixed displacement on a region of the casing and we will explore the consequent stress fields produced in the casing. One subsequent question to ask is how effective this plug will be in closing off the well. A key physical parameter here is the friction formed between the plug and the casing and this ought perhaps to be determined by some kind of pull-out test. Assuming we have a sensible value for this parameter, our analysis will give a connection between the size of the expanded plug and its expanded radius versus the cased wellbore radius (i.e. the displacement into the casing/rock) together with the pressure differential such a plug might be expected to withstand. In principle our theory (or a slightly more complicated one) could give an idea of where associated fractures may occur in the formation. We do make the simplifying assumption that the geometry is axisymmetric which is a reasonable starting assumption. We take the $x$ coordinate to be in the axial direction and $r$ to be the radial coordinate and then write the equations in terms of Love's stress function $\varphi(r, x)$ (Love [7]) which is to satisfy the differential equation

$\Delta^{2} \varphi(r, x)=\left(\frac{\partial^{2}}{\partial r^{2}}+\frac{1}{r} \frac{\partial}{\partial r}+\frac{\partial^{2}}{\partial x^{2}}\right)^{2} \varphi(r, x)=0$,

where

$\Delta=\frac{\partial^{2}}{\partial r^{2}}+\frac{1}{r} \frac{\partial}{\partial r}+\frac{\partial^{2}}{\partial x^{2}}$, 
and

$$
\begin{aligned}
\sigma_{r} & =\frac{\partial}{\partial x}\left[v \Delta-\frac{\partial^{2}}{\partial r^{2}}\right] \varphi(r, x), \\
\sigma_{x} & =\frac{\partial}{\partial x}\left[(2-v) \Delta-\frac{\partial^{2}}{\partial x^{2}}\right] \varphi(r, x), \\
\sigma_{\vartheta} & =\frac{\partial}{\partial x}\left[v \Delta-\frac{1}{r} \frac{\partial}{\partial r}\right] \varphi(r, x), \\
\sigma_{r x} & =\frac{\partial}{\partial r}\left[(1-v) \Delta-\frac{\partial^{2}}{\partial x^{2}}\right] \varphi(r, x),
\end{aligned}
$$

where $v$ denotes Poisson's ratio and $\theta$ is the azimuthal angle. The radial and axial displacements appear as

$u=-\frac{(1+v)}{E} \frac{\partial^{2}}{\partial r \partial x} \varphi(r, x)$

$v=\frac{(1+v)}{E}\left[(1-2 v) \Delta+\frac{\partial^{2}}{\partial r^{2}}+\frac{1}{r} \frac{\partial}{\partial r}\right] \varphi(r, x)$.

We need to solve these equations with the appropriate boundary conditions on the wellbore wall which for the cased hole is the inside of the casing. We treat the simplest case here although the methods we use can be adapted to more complicated cases at the expense of some algebra. The first major approximation is to treat the casing and the rock as one homogenised medium which means the moduli of this medium are somewhere between that of steel and rock. As discussed above, we could allow for a radial extent of casing and an infinite extent of rock without a major change in our method although it would be algebraically much more complicated and involve more physical parameters. We will discuss in Appendix how we might choose our homogenised medium. We solve Eq. (1) by taking a Fourier transform over $x$ as

$\Phi(r, \zeta)=\int_{-\infty}^{\infty} \exp (\mathrm{i} \zeta x) \varphi(r, x) \mathrm{d} x$.

The solution which tends to zero as $r$ tends to infinity can be written as

$\Phi(r, \zeta)=A_{1}(\zeta) K_{0}(|\zeta| r)+A_{2}(\zeta)|\zeta| r K_{1}(|\zeta| r)$,

where $K_{0}$ and $K_{1}$ are Bessel functions. Applying the Fourier transform to the stress and displacement equations gives

$$
\begin{aligned}
\Sigma_{r} & =\mathrm{i} \zeta^{3}\left[A_{1}\left(K_{0}+K_{1} / r_{1}\right)-A_{2}\left((1-2 v) K_{0}-r_{1} K_{1}\right)\right], \\
\Sigma_{r x} & =|\zeta|^{3}\left[-A_{1} K_{1}+A_{2}\left((2-2 v) K_{1}-r_{1} K_{0}\right)\right], \\
\Sigma_{\vartheta} & =-\mathrm{i} \zeta^{3}\left[A_{1} \frac{K_{1}}{r_{1}}+A_{2}(1-2 v) K_{0}\right], \\
\Sigma_{x} & =-\mathrm{i} \zeta^{3}\left[A_{1} K_{0}-A_{2}\left(2(2-v) K_{0}-r_{1} K_{1}\right)\right], \\
U & =\frac{1+v}{E} \mathrm{i} \zeta|\zeta|\left[-A_{1} K_{1}-A_{2} r_{1} K_{0}\right], \\
V & =\frac{1+v}{E} \zeta^{2}\left[A_{1} K_{0}-A_{2}\left(4(1-v) K_{0}-r_{1} K_{1}\right)\right] .
\end{aligned}
$$

In the above equations capital letters are used to designate the Fourier transform of the appropriate stresses and displacements, the argument of the Bessel functions being $r_{1}=r|\zeta|$. We proceed to find a Green's function which satisfies the boundary conditions on the wellbore wall which is at $r=a$. These conditions are

$\sigma_{r x}=0$,

on $r=a$ for all $x$ and

$\sigma_{r}=\delta(x)$, 
on $r=a$ for all $x$. Thus the Fourier transformed boundary conditions become

$\Sigma_{r x}=0, \quad \Sigma_{r}=1$,

on $r=a$. The arguments of the Bessel functions are thus $r_{1}=a|\zeta|$ for these boundary conditions. We thus have to solve the following equation for $A_{1}$ and $A_{2}$,

$-A_{1} K_{1}\left(r_{1}\right)+A_{2}\left((2-2 v) K_{1}\left(r_{1}\right)-r_{1} K_{0}\left(r_{1}\right)\right)=0$.

Solving this equation gives with $r_{1}=a|\zeta|$

$A_{1}=A_{2}\left[(2-2 v)-a|\zeta| \frac{K_{0}(a|\zeta|)}{\left.K_{1}(a \mid \zeta) \mid\right)}\right]$,

which when substituted into

$\mathrm{i} \zeta^{3}\left[A_{1}\left(K_{0}\left(r_{1}\right)+\frac{K_{1}\left(r_{1}\right)}{r_{1}}\right)-A_{2}\left((1-2 v) K_{0}\left(r_{1}\right)-r_{1} K_{1}\left(r_{1}\right)\right)\right]=1$,

gives $A_{2}$,

$A_{2}=\frac{\mathrm{i} a K_{1}(a|\zeta|)}{\zeta|\zeta|} \frac{1}{\left.\left[(a \zeta)^{2}\left(K_{0}(a|\zeta|)^{2}-K_{1}(a \mid \zeta) \mid\right)^{2}\right)-2(1-v) K_{1}(a|\zeta|)^{2}\right]}$.

Note that $A_{2}$ is odd in $\zeta$ and hence so is $A_{1}$. Once $A_{1}$ and $A_{2}$ are found from these equations, it is straightforward to evaluate

$U(r, \zeta)=\frac{1+v}{E} \mathrm{i} \zeta|\zeta|\left[-A_{1} K_{1}\left(r_{1}\right)-A_{2} r_{1} K_{0}\left(r_{1}\right)\right]$,

which becomes on $r=a$,

$U(a, \zeta)=2 a \frac{\left(1-v^{2}\right)}{E} \frac{K_{1}(a|\zeta|)^{2}}{\left.\left[(a \zeta)^{2}\left(K_{0}(a|\zeta|)^{2}-K_{1}(a \mid \zeta) \mid\right)^{2}\right)-2(1-v) K_{1}(a|\zeta|)^{2}\right]}$.

This is the Fourier transform of the radial displacement anywhere and in particular on the casing boundary $r=a$, i.e. $r_{1}=|\zeta| a$. Inverting thus gives

$u(r, x)=\frac{1}{2 \pi} \int_{-\infty}^{\infty} \exp (-\mathrm{i} \zeta x) U(r, \zeta) \mathrm{d} \zeta$.

We note that $U(r, \zeta)$ is an even function of $\zeta$ because of the properties of $A_{1}$ and $A_{2}$. This Green's function was generated by a delta function loading at $x=0$. We now move the origin to $x=x^{\prime}$ and multiply by an unknown radial stress $\sigma_{r}\left(a, x^{\prime}\right)$. Finally we get the integral representation

$u(r, x)=\int_{-l}^{l} \sigma_{r}\left(a, x^{\prime}\right)\left(\frac{1}{2 \pi} \int_{-\infty}^{\infty} \exp \left(-\mathrm{i} \zeta\left(x-x^{\prime}\right)\right) U(r, \zeta) \mathrm{d} \zeta\right) \mathrm{d} x^{\prime}$.

We integrate over $x^{\prime}$ from $-l$ to 1 since we designate this as the contact region. The expression $\sigma_{r}\left(a, x^{\prime}\right)$ is still unknown and must be determined from the integral equation which follows from setting $u(a, x)$ equal to the contact displacement due to the expansion into the casing. This contact takes place over the region $-l<x<l$ at $r=a$. Once we have solved this integral equation for the unknown contact stresses, we can evaluate the stress and displacement fields everywhere. Because $U(r, \zeta)$ is an even function of $\zeta$, we can simplify the above integral representation as

$u(r, x)=\int_{-l}^{l} \sigma_{r}\left(a, x^{\prime}\right)\left(\frac{1}{\pi} \int_{0}^{\infty} \cos \left(\zeta\left(x-x^{\prime}\right)\right) U(r, \zeta) \mathrm{d} \zeta\right) \mathrm{d} x^{\prime}$.

We also anticipate that both $u(r, x)$ and $\sigma_{r}(a, x)$ will be even functions of $x$ which will simplify the integral representation further. Although our method can deal with more complicated displacements in the contact region, we assume $u(a, x)=u_{0}$ a constant for $-l<x<l$ and approximate the normal stress variation by

$\sigma_{r}(a, x)=B / \sqrt{ }\left(l^{2}-x^{2}\right)$. 
Note that this stress field is singular near the ends of the contact region. This can be shown to be a consequence of the contact boundary conditions and would also appear if we solved the integral equation numerically for all but special contact boundary conditions designed to remove this singularity. Arguments to support this contention can be made by approaching closely the ends of the contact region and scaling coordinates so that close to each end it looks locally plane. Alternatively note that $\int_{0}^{\infty} \cos (\zeta x)(\operatorname{Si}(\zeta) / \zeta) \mathrm{d} \zeta$ is proportional to $\log (\mathrm{x})$ as $x$ tends to zero and the ratio $(\operatorname{Si}(\zeta) / \zeta) / U(r, \zeta)$ is finite as $\zeta$ tends to infinity so we can infer that the integral equation over $x^{\prime}$ has a dominant logarithmic singularity hence the assumed form $\sigma_{r}(a, x)=B / \sqrt{ }\left(l^{2}-x^{2}\right)$. This is not our main concern here because we are interested in the average stress due to the contact conditions. Changing the order of integration the integral equation now becomes

$u_{0}=\frac{1}{\pi} \int_{0}^{\infty} U(a, \zeta)\left(\int_{-l}^{l} \sigma_{r}\left(a, x^{\prime}\right) \cos \left(\zeta\left(x-x^{\prime}\right)\right) \mathrm{d} x^{\prime}\right) \mathrm{d} \zeta$,

and using the above expression for $\sigma_{r}(a, x)$ it is convenient to use the substitutions $x=l \cos (t)$ and $x^{\prime}=l \cos \left(t^{\prime}\right)$ and integrate over $t$ and $t^{\prime}$ from zero to $\pi$. Thus the integral equation is satisfied approximately. A numerical method using Tshebychev polynomials could have been used here but is not essential for our purpose. We find

$u_{0}=B \int_{0}^{\infty} U(a, \zeta) J_{0}(l \zeta)^{2} \mathrm{~d} \zeta$

Finally if we substitute $l \zeta=\zeta_{1}$ in the above integral we see that $B$ is proportional to $\left(2 l u_{0} E\right) /\left(2 a\left(1-v^{2}\right)\right)$ and inversely proportional to an integral which is weakly dependent on $v$ and depends strongly on $a / l$.

\section{A simple force balance for plug placement}

From the above analysis we have calculated the compressive contact stress $\sigma_{r}(a, x)$ on the wall due to the plug expanding into it. This average stress (force per unit area) is just $(B \pi) /(2 l)$. To estimate the resistance to movement of the plug due to the differential pressure from bottom to top of the plug, we need some knowledge of the friction coefficient of the bond between the expanded plug and the wall (whether it be casing or rock). This could perhaps be obtained by an appropriate pull-out test. If we assume this is known and we call it $\eta$, then the net resistance to movement due to this friction is $\eta(B \pi)(2 \pi a)$ where $a$ and $2 l$ are the actual radius of the wellbore and length of the contact region. This has to be balanced with the force trying to move the plug which will be $\left(P_{2}-P_{1}\right) \pi a^{2}$ where $P_{2}-P_{1}$ is the difference in pressure between the bottom and top of the plug. Balancing these forces and substituting for $B$ gives

$\frac{2 \pi E \eta}{\left(1-v^{2}\right)} \frac{u_{0}}{a} \geq\left(P_{2}-P_{1}\right) I$

where $I$ is given by

$I=-\int_{0}^{\infty} \frac{a_{1} J_{0}(\zeta)^{2} K_{1}\left(a_{1}|\zeta|\right)^{2}}{\left.\left[\left(a_{1} \zeta\right)^{2}\left(K_{0}\left(a_{1}|\zeta|\right)^{2}-K_{1}\left(a_{1} \mid \zeta\right) \mid\right)^{2}\right)-2(1-v) K_{1}\left(a_{1}|\zeta|\right)^{2}\right]} \mathrm{d} \zeta$

with $a_{1}=a / l$. When one plots the integral $I$ against $a_{1}$, we see as might be expected that it increases as $a_{1}$ increases implying that the effect of the differential pressure decreases as the ratio of contact length to radius increases. In Eq. (32) $E$ is an effective medium value of Young's modulus taking into account the casing thickness. Obviously this lies between the Young's modulus of the casing and that of the rock. An expression for an effective shear modulus is given in Appendix. Also the correct casing geometry case can be evaluated by the methods of this note but is algebraically more complicated, where $u_{0} / a$ is the dimensionless expansion into the casing, and the major unknown is $\eta$ the friction coefficient. The detailed stress field could also be evaluated if required. Furthermore the analysis can be extended to multiple contact regions in a straightforward way. 


\section{4 'Soft' contact problems and displacement-dependent surface interactions}

Some years ago Atkinson [8] considered displacement in a penny-shaped crack under displacement-dependent internal stresses, and the result was a non-linear integral equation which was treated numerically. In that work the motivation was that fibres might bridge the crack and give rise to a crack boundary condition that relates the stress on the crack faces to the crack opening that represents the bridging action of the fibres as the crack opens. However the method proposed was designed to apply to any non-linear boundary condition acting on the crack faces. There are of course many other situations that could give rise to a non-linear boundary condition. Hughes and White [9] have derived non-linear integral equations over a flat surface as a model of a 'soft' contact theory in colloid science. Here we could consider the effect of such boundary conditions in the contact region by means of specifying $\sigma_{r}(a, x)$ as a known function of $u(a, x)$ for $-l<x<l$. Using this in Eq. (28) when $r=a$ gives a non-linear integral equation for the displacement $u(a, x)$ over the contact region which could also be treated by the methods used by Hughes and White.

Open Access This article is distributed under the terms of the Creative Commons Attribution 4.0 International License (http:// creativecommons.org/licenses/by/4.0/), which permits unrestricted use, distribution, and reproduction in any medium, provided you give appropriate credit to the original author(s) and the source, provide a link to the Creative Commons license, and indicate if changes were made.

\section{Appendix: A simple homogenisation}

We imagine a two-dimensional situation in which a radial displacement is applied at an inner boundary of radius $r=a$ and casing between $r=a$ and $r=b$. We have continuity of stress and displacement at boundary $r=b$ together with Lame constants $\lambda_{1}$ and $\mu_{1}$ in the casing $a<r<b$ with the same constants with subscript 2 in the rock for $r>b$. The $\Sigma_{r}$ stress is matched at the boundary $r=a$ for a homogeneous medium $r \geq a$ and homogeneous modulus $\mu$ where

$\mu=\frac{\mu_{1}\left(3 \lambda_{1}+2 \mu_{1}+4 \mu_{2}\right)-4\left(\mu_{1}-\mu_{2}\right)\left(3 \lambda_{1}+2 \mu_{1}\right)(a / b)^{3}}{\left(3 \lambda_{1}+2 \mu_{1}+4 \mu_{2}\right)+4\left(\mu_{1}-\mu_{2}\right)(a / b)^{3}}$.

Note for $\mu_{1}>\mu_{2}$ we have $\mu_{2}<\mu<\mu_{1}$ with equality when $\mu_{1}=\mu_{2}$.

\section{References}

1. Tranter CJ, Craggs JW (1944) The stress distribution in a long circular cylinder when discontinuous pressure is applied to the curved surface. Lond Edinb Dublin Philos Mag J Sci 36(255):241-250

2. Atkinson C, Desroches J, Eftaxiopoulos DA, Thiercelin M (2001) Wellbore stresses induced by the nonlinear deformation of an inflatable packer. J Eng Math 41:305-327

3. Kydoniefs AD (1970) Finite axisymmetric deformations of an initially cylindrical membrane reinforced with inextensible cords. Q J Mech Appl Math 23:481-488

4. Atkinson C, Peltier B (1993) The finite deformation of a reinforced packer, membrane theory. J Eng Math 27:443-454

5. Selvadurai AP, Suvorov AP (2018) On the development of instabilities in an annulus and a shell composed of a poro-hyperelastic material. Proc R Soc A 474:20180239

6. Eftaxiopoulos DA, Atkinson C (2005) A nonlinear, anisotropic and axisymmetric model for balloon angioplasty. Proc R Soc A 461:1097-1128

7. Love AEH (1927) A treatise on the mathematical theory of elasticity. Cambridge University Press, Cambridge

8. Atkinson C (1970) An iterative scheme for solving problems relating to cracks opening under a displacement-dependent internal stress. Int J Fract Mech 6:193-198

9. Hughes BD, White LR (1979) 'Soft'contact problems in linear elasticity. Q J Mech Appl Math 32:445-471

Publisher's Note Springer Nature remains neutral with regard to jurisdictional claims in published maps and institutional affiliations. 